\title{
The Effect of Non-penicillin Antibiotic Regimens on Neonatal Outcomes in Preterm Premature Rupture of Membranes
}

\author{
Anne M. Siegel, MD ${ }^{1}$ Robert Phillips Heine, MD ${ }^{1} \quad$ Sarah K. Dotters-Katz, MD ${ }^{1}$ \\ ${ }^{1}$ Division of Maternal Fetal Medicine, Department of Obstetrics and \\ Gynecology at Duke University Hospital, Durham, North Carolina \\ Address for correspondence Anne M. Siegel, MD, Division of Maternal \\ Fetal Medicine, Department of Obstetrics and Gynecology, Duke \\ University, Address: 2608 Erwin Road, Suite 210, Durham, NC 27705 \\ Am J Perinatol Rep 2019;9:e67-e71. \\ (e-mail: anne.siegel@duke.edu).
}

\begin{abstract}
Objective A 7-day course of a penicillin (PCN) and macrolide is standard of care (SAR) in preterm premature rupture of membranes (PPROM). Data regarding alternative antibiotic regimens are limited. We sought to assess the impact of non-PCN regimens on neonatal outcomes.

Study Design Secondary analysis of randomized controlled trial of antenatal magnesium sulfate. Singleton, nonanomalous pregnancies complicated by PPROM at $>24$ weeks of gestation receiving the SAR were compared with women receiving a non- $\beta$ lactam regimen and a macrolide (NPCR). Primary outcome was a neonatal composite. Secondary outcomes included pregnancy latency, endometritis, and chorioamnionitis. Results A total of 949 women met inclusion criteria; 821(56\%) received the SAR and $128(8.8 \%)$ received NPCR. Adjusted models did not demonstrate worse outcomes (AOR [adjusted odds ratio] $=0.50 ; 95 \% \mathrm{Cl}$ [confidence interval]: 0.22-1.11). Neonates

\section{Keywords}

- preterm premature rupture of membranes

- antibiotics

- penicillin allergy

- obstetric infectious disease

receiving SAR were less likely to have bronchopulmonary dysplasia (BPD; $p=0.03$ ) but more likely to have severe necrotizing enterocolitis (sNEC; $p=0.04$ ). Risk for chorioamnionitis and median latency did not differ between groups but women receiving the SAR were less likely to get endometritis (AOR $=0.35 ; 95 \% \mathrm{Cl}: 0.14-0.91$ ). Conclusions In this cohort, receiving NPCR in the setting of PPROM did not impact the overall risk of adverse neonatal outcomes or latency, but did increase the risk of endometritis. Alterations in individual neonatal morbidities suggest follow-up studies are needed.
\end{abstract}

Preterm premature rupture of membranes (PPROM) effects approximately 3\% of all pregnancies in the United States. ${ }^{1}$ Pregnancies complicated by PPROM pose maternal risks including complications from chorioamnionitis, postpartum infections, and placental abruption, while their neonates face complications related to preterm birth including respiratory distress, necrotizing enterocolitis (NEC), intraventricular hemorrhage (IVH), sepsis, and death. Additionally, fetuses exposed to intrauterine inflammation in PPROM have an increased risk of cerebral palsy. ${ }^{2}$

received

November 12, 2018 accepted after revision January 9, 2019
DOI https://doi.org/

10.1055/s-0039-1683378. ISSN 2157-6998.
Antibiotics in PPROM reduce neonatal morbidity from sepsis, pneumonia, respiratory distress, and necrotizing enterocolitis. ${ }^{3-7}$ Additionally, antibiotics prolong pregnancy latency and reduce maternal infectious morbidity from chorioamnionitis. ${ }^{3,5,7}$ Given the body of literature on the benefits of antibiotic use in PPROM, the standard of care is a 7-day course of a penicillin (PCN) and macrolide for all women with this complication. ${ }^{3}$

One poorly understood caveat to this is the appropriate management strategy of women with PCN allergy, a common
Copyright $\odot 2019$ by Thieme Medical Publishers, Inc., 333 Seventh Avenue, New York, NY 10001, USA. Tel: +1(212) 584-4662.
License terms

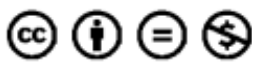


drug class allergy. PCN allergy is reported in up to $8 \%$ of individuals accessing care in the United States. ${ }^{8}$ Although the majority of patients with a self-reported PCN allergy do not have a true allergy, ${ }^{9}$ physicians may be reticent to administer a $\beta$-lactam antibiotic in this setting. No consistent recommendations regarding the appropriate antibiotic regimen to use in cases of PPROM in a patient with a PCN allergy exist. Also, some physicians may prefer non- $\beta$-lactam regimens for management of PPROM. However, data regarding the impact of alternative antibiotic regimen on neonatal morbidity and mortality as well as maternal infectious morbidity are unavailable. The objective of this study was to assess the impact of a non- $\beta$-lactam antibiotic regimen in expectant management of PPROM on neonatal morbidity and mortality. Additionally, we sought to describe impact of non- $\beta$-lactam antibiotic regimen on pregnancy latency and maternal infectious outcomes.

\section{Methods}

This study is a secondary analysis of the Eunice Kennedy Shriver National Institute of Child Health and Human Development, Maternal-Fetal Medicine Units Network, beneficial effects of antenatal magnesium sulfate (BEAM) randomized, placebo controlled, and multicenter trial of antenatal magnesium sulfate for the prevention of cerebral palsy. ${ }^{10}$ Duke University institutional review board determined the study to be exempt under protocol Pro00065517. We included singleton, nonanomalous pregnancies complicated by PPROM at or beyond $24^{0 / 7}$ weeks of gestation and delivery at less than $35^{0 / 7}$ weeks. The gestational age of 35 weeks was selected to minimize exclusion of women with PPROM who were induced starting at 34 weeks. We excluded multiple gestations, pregnancies complicated by chromosomal abnormalities, pregnancies where no antibiotics were administered, and those subjects with a missing date or time of rupture or delivery.

Women receiving the gold standard antibiotic regimen $\beta$ lactam and macrolide were compared with women receiving a non- $\beta$-lactam and macrolide regimen. $\mathrm{PCN}$ and cephalosporin antibiotic regimens were both included in the $\beta$-lactam group given their similar mechanism of action and similar hypersensitivity response. The primary outcome was a neonatal composite of severe necrotizing enterocolitis (NEC; defined as grade 2 or 3 ), severe intraventricular hemorrhage (defined as grade 4), periventricular leukomalacia (PVL), bronchopulmonary dysplasia (BPD), neonatal death prior to hospital discharge, sepsis, and cerebral palsy. Our secondary outcomes included all the components of the composite, pregnancy latency, and maternal endometritis and chorioamnionitis. Pregnancy latency was defined as the interval of time in days from membrane rupture to delivery.

The characteristics and outcomes of subjects receiving the different antibiotic regimens were compared using KruskalWallis, Chi-squared or Fisher's exact test as appropriate. Multivariable logistic regression models for the outcomes of interest were then developed to estimate the true effect of the $\beta$-lactam and macrolide versus non- $\beta$-lactam antibiotic regimen. Covariates in the regression model included those significant in the bivariate analysis and those with known clinical associations to the outcome of interest. Backward stepwise regression was used with covariates with $p<0.20$ were retained in final models. Variables in original model for neonatal composite included maternal age, marital status, maternal drug use, maternal tobacco use, mode of delivery, neonatal gender, neonatal birth weight, gestational age at birth, neonatal magnesium exposure, corticosteroids, group B streptococcus status, chorioamnionitis, and diabetes. The same statistical analysis was performed for the secondary analysis. STATA 13.1 (StataCorp, College Station, TX) was used to perform all analyses.

\section{Results}

Of 2,444 pregnancies identified in the original BEAM trial, 949 women were included in the primary analysis (-Fig. 1); 821 (86.5\%) patients received a $\beta$-lactam and macrolide and 128 (13.5\%) received a non- $\beta$-lactam and macrolide. Within the non- $\beta$-lactam and macrolide group, 82 of the 128 women (64.1\%) received clindamycin. Compared with women who received the $\beta$-lactam and macrolide antibiotic regimen, women who received the non- $\beta$-lactam and macrolide were older $(p=0.03)$, more likely to be Caucasian $(p<0.01)$, married $(p<0.01)$, and less likely to use illicit drugs $(p=0.01 ;-$ Table1). No differences in receipt of magnesium, corticosteroids, or tocolytics were noted (-Table $\mathbf{2}$ ). Women receiving the gold standard antibiotic regimen had membrane rupture at a later median gestational age, 28.3 (IQR [interquartile range]: 26.3-30.3) weeks versus 27.3 (IQR: 25.3-30.0) weeks ( $p=0.03$ ). However, no differences in rates of spontaneous labor, Cesarean delivery, gestational age at delivery, birth weight, gender of newborn, or 5 minute Apgar's score between groups were noted ( $\mathbf{- T a b l e ~} \mathbf{2}$ ).

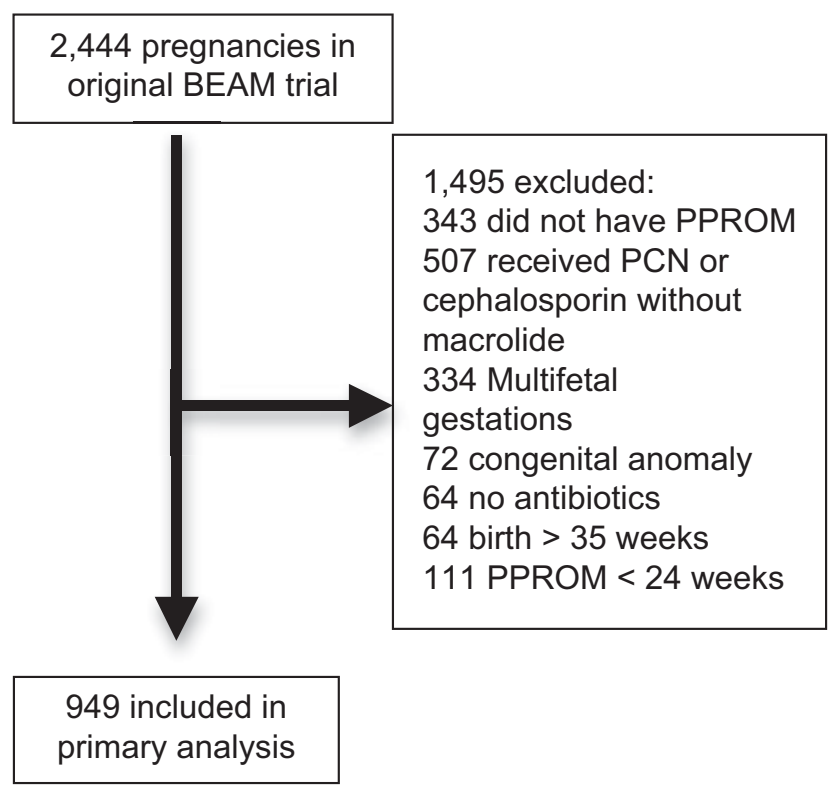

Fig. 1 Flow diagram of patients meeting inclusion and exclusion criteria. 
Table 1 Demographic data of women receiving a $\beta$-lactam and macrolide compared with a non- $\beta$-lactam regimen after preterm premature rupture of membranes

\begin{tabular}{|c|c|c|c|}
\hline & $\begin{array}{l}\beta \text {-Lactam and } \\
\text { macrolide } \\
\text { regimen } \\
n=821(\%)\end{array}$ & $\begin{array}{l}\text { Non- } \beta \text {-lactam } \\
\text { regimen } \\
n=128(\%)\end{array}$ & $p$-Value \\
\hline Median age, (IQR) & $26(21,31)$ & $27(22,33)$ & 0.03 \\
\hline \multicolumn{3}{|l|}{ Race } & \multirow[t]{5}{*}{$<0.01$} \\
\hline Caucasian & $442(53.8)$ & $47(36.7)$ & \\
\hline Black & $297(36.2)$ & 69 (53.9) & \\
\hline Hispanic & 65 (7.9) & $11(8.6)$ & \\
\hline Other & $17(2.1)$ & $1(0.8)$ & \\
\hline Hispanic & 65 (7.9) & $11(8.6)$ & 0.79 \\
\hline $\begin{array}{l}\text { High school } \\
\text { education or less }\end{array}$ & $586(71.4)$ & $87(68.0)$ & 0.43 \\
\hline Married & $331(40.3)$ & $74(57.8)$ & $<0.01$ \\
\hline Obese (BMI > 30) & $258(31.4)$ & $39(30.5)$ & 0.83 \\
\hline Multiparous & $538(65.5)$ & $89(69.5)$ & 0.37 \\
\hline Prior PTB & $252(30.7)$ & $30(23.4)$ & 0.10 \\
\hline Smoking & $287(35.0)$ & $42(32.8)$ & 0.64 \\
\hline Drugs use & $120(14.6)$ & $7(5.5)$ & 0.01 \\
\hline GBS positive & $470(57.3)$ & $78(60.9)$ & 0.43 \\
\hline Diabetes & $39(4.8)$ & $7(5.5)$ & 0.73 \\
\hline Any prenatal care & 752 (91.6) & $119(93.0)$ & 0.60 \\
\hline
\end{tabular}

Abbreviations: BMI, body mass index; GBS, group B strep; IQR, interquartile range; $B E A M$, beneficial effects of antenatal magnesium sulfate; PCN, penicillin; PPROM, preterm premature rupture of membranes; PTB, preterm birth.

The primary neonatal composite outcome occurred in $28.3 \%$ of the $\beta$-lactam and macrolide group and $35.2 \%$ of the non- $\beta$-lactam and macrolide group $(p=0.11)$. When the individual components of the neonatal composite were analyzed, with the exception of severe necrotizing enterocolitis, neonates receiving the $\beta$-lactam and macrolide group trended toward a reduction in adverse outcomes, with bronchopulmonary dysplasia reaching clinical significance $(p=0.03 ;-$ Table 3$)$. With regards to severe necrotizing enterocolitis, the inverse was seen $(p=0.04)$. In adjusted analyses, no difference between groups for the neonatal composite (adjusted odds ratio $[\mathrm{AOR}]=0.50$; 95\% confidence interval [CI]: 0.22-1.11) was found when adjusting for maternal age, race, maternal drug use, neonatal gender, gestational age at birth, birth weight, magnesium exposure, clindamycin exposure, and corticosteroids

Regarding maternal outcomes, risk of chorioamnionitis and endometritis did not differ, nor did the median latency (6.9 days [IQR: 3.4-13.5] vs. 7.0 days [IQR: 3.315.4]; - Table 3). After controlling for confounders, the risk for chorioamnionitis remained nonsignificant (-Table 4). However, women receiving the gold standard antibiotic regimen were less likely to get endometritis, $(\mathrm{AOR}=0.35$; 95\% CI: 0.14-0.91).
Table 2 Delivery and neonatal characteristics of women receiving a $\beta$-lactam and macrolide compared with a non- $\beta$ lactam regimen after preterm premature rupture of membranes

\begin{tabular}{|l|l|l|l|}
\hline & $\begin{array}{l}\boldsymbol{\beta} \text {-lactam and } \\
\text { macrolide } \\
\text { regimen } \\
\boldsymbol{n}=\mathbf{8 2 1}(\%)\end{array}$ & $\begin{array}{l}\text { Non- } \boldsymbol{\beta} \\
\text { lactam } \\
\text { regimen } \\
\boldsymbol{n}=\mathbf{1 2 8}(\%)\end{array}$ & $p$-Value \\
\hline $\begin{array}{l}\text { Median gestational } \\
\text { age at membrane } \\
\text { rupture, wk, (IQR) }\end{array}$ & $\begin{array}{l}28.3 \\
(26.3,30.3)\end{array}$ & $\begin{array}{l}27.3 \\
(25.3,30.0)\end{array}$ & 0.03 \\
\hline $\begin{array}{l}\text { Any magnesium } \\
\text { exposure }\end{array}$ & $147(17.9)$ & $27(21.1)$ & 0.39 \\
\hline Corticosteroids & $813(99.0)$ & $125(97.7)$ & 0.18 \\
\hline Tocolytic exposure & $20(2.4)$ & $0(0.0)$ & 0.07 \\
\hline $\begin{array}{l}\text { Exposure to } \\
\text { clindamycin }\end{array}$ & $0(0.0)$ & $82(64.1)$ & $<0.01$ \\
\hline Spontaneous labor & $434(52.9)$ & $69(53.9)$ & 0.84 \\
\hline $\begin{array}{l}\text { Median gestational } \\
\text { age at delivery, } \\
\text { wk, (IQR) }\end{array}$ & $\begin{array}{l}30.1 \\
(27.9,31.7)\end{array}$ & $\begin{array}{l}29.7 \\
(27.2,31.6)\end{array}$ & 0.08 \\
\hline Cesarean delivery & $306(37.3)$ & $52(40.6)$ & 0.47 \\
\hline $\begin{array}{l}\text { Median birth } \\
\text { weight, g, (IQR) }\end{array}$ & $\begin{array}{l}1,395 \\
(1,037,1,730)\end{array}$ & $\begin{array}{l}1,355 \\
(989-1,731)\end{array}$ & 0.48 \\
\hline Male infant & $435(53.0)$ & $60(46.9)$ & 0.20 \\
\hline $\begin{array}{l}\text { 5-minute Apgar's } \\
\text { score < 7 }\end{array}$ & $131(16.0)$ & $23(18.0)$ & 0.57 \\
\hline
\end{tabular}

Abbreviation: IQR, interquartile range.

\section{Discussion}

Among women with pregnancies affected by PPROM, receiving a non- $\beta$-lactam and macrolide containing antibiotic regimen was associated with increased risk of endometritis. Neonates born to women who received the gold standard antibiotic regimen of a $\beta$-lactam and macrolide were less likely to have bronchopulmonary dysplasia but more likely to have severe necrotizing enterocolitis.

Our findings suggest improved neonatal outcomes with administration of $\beta$-lactam antibiotics in PPROM those are supported by previous research. The current standard of care for antibiotic administration in PPROM was established based on the results from a large, multicenter, randomized controlled trial evaluating use of ampicillin and erythromycin on a composite neonatal outcome. ${ }^{3}$ In that study, a decrease in composite neonatal morbidity, respiratory distress, necrotizing enterocolitis, sepsis, pneumonia, patent ductus arteriosus, bronchopulmonary dysplasia, and hyperbilirubinemia with $\beta$ lactam and macrolide antibiotic administration was seen. ${ }^{3}$ Our study, combined with many other studies, has demonstrated improvement in neonatal outcomes in PPROM with administration of a $\beta$-lactam antibiotic. ${ }^{4-6,11}$ However, Mercer et $\mathrm{al}^{3}$ excluded women with allergy to PCN or erythromycin from their study. Thus, our study is unique in its aim to describe the effect of non- $\beta$-lactam antibiotic regimens in PPROM used in the setting of a PCN allergy. 
Table 3 Maternal and neonatal outcomes for women receiving a $\beta$-lactam and macrolide compared with a non- $\beta$-lactam regimen after preterm premature rupture of membranes

\begin{tabular}{|c|c|c|c|}
\hline & $\begin{array}{l}\beta \text {-lactam and } \\
\text { macrolide } \\
\text { regimen } \\
n=821(\%)\end{array}$ & $\begin{array}{l}\text { Non- } \beta \text {-lactam } \\
\text { and macrolide } \\
\text { regimen } \\
n=128(\%)\end{array}$ & $p$-Value \\
\hline \multicolumn{4}{|l|}{ Maternal } \\
\hline $\begin{array}{l}\text { Median latency } \\
\text { until delivery, } \\
\text { d (IQR) }\end{array}$ & $\begin{array}{l}6.9 \\
(3.4,13.5)\end{array}$ & $\begin{array}{l}7.0 \\
(3.3,15.4)\end{array}$ & 0.74 \\
\hline Endometritis & $49(6.0)$ & $12(9.4)$ & 0.14 \\
\hline Chorioamnionitis & $94(11.5)$ & $18(14.1)$ & 0.39 \\
\hline \multicolumn{4}{|l|}{ Neonatal } \\
\hline Composite & $232(28.3)$ & $45(35.2)$ & 0.11 \\
\hline Sepsis & $108(13.3)$ & $24(18.8)$ & 0.10 \\
\hline Death in NICU & $29(3.6)$ & $6(4.7)$ & 0.53 \\
\hline Severe IVH & $13(1.6)$ & $3(2.4)$ & 0.54 \\
\hline PVL & $12(1.5)$ & $4(3.2)$ & 0.18 \\
\hline BPD & $128(15.7)$ & $30(23.4)$ & 0.03 \\
\hline Severe NEC & $38(4.7)$ & $1(0.8)$ & 0.04 \\
\hline $\begin{array}{l}\text { Any cerebral } \\
\text { palsy }\end{array}$ & $29(3.7)$ & $8(6.5)$ & 0.14 \\
\hline
\end{tabular}

Abbreviations: BPD, bronchopulmonary dysplasia; IQR, interquartile range; IVH, intraventricular hemorrhage; NEC, necrotizing enterocolitis; NICU, Neonatal intensive care unit; PVL, periventricular leukomalacia.

Table 4 Adjusted odds ratio of maternal and neonatal outcomes for women receiving a $\beta$-lactam and macrolide compared with a non- $\beta$-lactam regimen

\begin{tabular}{|l|l|l|l|}
\hline & $\begin{array}{l}\text { Odds } \\
\text { ratio }\end{array}$ & $\begin{array}{l}\text { 95\% confidence } \\
\text { interval }\end{array}$ & $p$-Value \\
\hline Neonatal Composite $^{\mathrm{a}}$ & 0.50 & $0.22-1.11$ & 0.09 \\
\hline Chorioamnionitis $^{\mathrm{b}}$ & 0.75 & $0.43-1.31$ & 0.31 \\
\hline Endometritis $^{\mathrm{c}}$ & 0.35 & $0.14-0.91$ & 0.03 \\
\hline
\end{tabular}

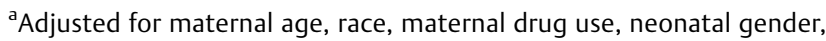
gestational age at birth, birth weight, magnesium exposure, clindamycin exposure, and corticosteroids.

${ }^{b}$ Adjusted for maternal age, race, group B strep status, and maternal smoking.

'Adjusted for clindamycin exposure, drug use, race, delivery route, and chorioamnionitis.

Our study is furthermore unique given the finding of decreased risk of endometritis in women receiving a $\beta$ lactam containing antibiotic regimen. Previous studies have shown that antibiotics in general decrease maternal chorioamnionitis and pregnancy latency in the setting PPROM. ${ }^{4,7,11}$ However, previous studies have not shown decreased rates of endometritis., ${ }^{5,6}$ However, other postpartum infectious morbidities have been shown to be more common among women receiving non- $\beta$-lactam regimens. ${ }^{12}$

The trend toward increasing severe necrotizing enterocolitis in the neonates receiving a $\beta$-lactam regimen in both analyses is not novel. Previous studies have demonstrated an increased risk of necrotizing enterocolitis with coamoxiclav, as well as with ampicillin. ${ }^{13,14}$ A plausible mechanism of action for the development of necrotizing enterocolitis in this population is alteration in the neonatal intestinal mircobiome. ${ }^{15}$ Several studies have shown that maternal antepartum antibiotic administration has altered neonatal intestinal bacterial colonization. ${ }^{16,17}$ Their newborn's intestinal mircobiome may also be altered and predispose the neonate to necrotizing enterocolitis.

The participants in the non- $\beta$-lactam group were exposed to alternative antibiotics and it is possible exposure to these antibiotics confounds our results. One of the most common antibiotics women in the non- $\beta$-lactam group received was clindamycin. Formulations of this antibiotic containing the preservative benzyl alcohol have been associated with adverse neonatal outcomes related to "gasping syndrome." 18,19 “Gasping syndrome" has been reported in premature infants exposed to clindamycin and results in multisystem organ failure and often death. ${ }^{19}$ Although we cannot determine the specific formulations that neonates in out cohort received, it is possible that women in the non- $\beta$-lactam group suffered adverse neonatal morbidities from their antibiotic exposure that were not quantified in our study.

We recognize other limitations to our study. As a retrospective cohort study, we are inherently limited to observational data, such as, we cannot assess causation. Additionally, given we performed a secondary analysis of the BEAM trial of antenatal magnesium sulfate for the prevention of cerebral palsy, we are limited in our analysis by the data that were collected for the primary analysis. For example, we do not have data on the timing of antibiotic administration in relation to PPROM, labor, and delivery; antibiotic dosing, total doses, and frequency of dosing. Our study design of a secondary analysis also limited our sample size and a post hoc power analysis demonstrated that the study was underpowered to show a difference. We detected at $24 \%$ increase in the primary outcome in the non- $\beta$-lactam and macrolide group. However, based on the ratio of women in $\beta$-lactam and macrolide group to non- $\beta$-lactam and macrolide group (roughly 7:1), we would need 2,767 women in the $\beta$-lactam group and 415 women in the non- $\beta$-lactam and macrolide group to detect a difference in the primary outcome. However, our findings are hypothesis generating and suggest need for further study. Also, we did have to exclude a large number of patients for either incomplete data or administration of a $\beta$-lactam antibiotic without a macrolide. Finally, we were unable to compare PCNs versus cephalosporins on maternal or neonatal outcomes given they were analyzed together as $\beta$-lactam antibiotics.

Our study also has several strengths. First, large sample size decreases risk of type 2 error. Also, because the original data were collected from multiple centers nationwide by trained data abstractors, the data represent a diverse patient population and the results are more likely to be generalized. Finally, as there is limited data available in the literature regarding the efficacy of a non- $\beta$-lactam regimen on women with PPROM, this study adds information for the clinician that is applicable in daily practice. 


\section{Conclusion}

In conclusion, women with PPROM receiving a $\beta$-lactam and macrolide were less likely to suffer from endometritis. Additionally, among neonates with PPROM the administration of a $\beta$-lactam antibiotics did not impact composite neonatal morbidity. However, alterations in individual neonatal morbidities suggest that follow-up studies are needed to further investigate the effects of non- $\beta$-lactam antibiotic regimens on pregnancies affected by PPROM. Given these associations, practitioners should appropriately use cephalosporins in patients with a nonsignificant PCN allergy and should consider PCN allergy testing in all pregnant women with significant allergies to optimize maternal and neonatal outcomes in pregnancies affected by PPROM in the setting of PCN allergy.

Conflicts of Interest

The authors report no conflicts of interest.

\section{References}

1 Waters TP, Mercer B. Preterm PROM: prediction, prevention, principles. Clin Obstet Gynecol 2011;54(02):307-312

2 Spinillo A, Capuzzo E, Stronati M, Ometto A, Orcesi S, Fazzi E. Effect of preterm premature rupture of membranes on neurodevelopmental outcome: follow up at two years of age. $\mathrm{Br} \mathrm{J}$ Obstet Gynaecol 1995;102(11):882-887

3 Mercer BM, Miodovnik M, Thurnau GR, et al; National Institute of Child Health and Human Development Maternal-Fetal Medicine Units Network. Antibiotic therapy for reduction of infant morbidity after preterm premature rupture of the membranes. A randomized controlled trial. JAMA 1997;278(12):989-995

4 Magwali TL, Chipato T, Majoko F, Rusakaniko S, Mujaji C. Prophylactic augmentin in prelabor preterm rupture of the membranes. Int J Gynaecol Obstet 1999;65(03):261-265

5 Christmas JT, Cox SM, Andrews W, Dax J, Leveno KJ, Gilstrap LC. Expectant management of preterm ruptured membranes: effects of antimicrobial therapy. Obstet Gynecol 1992;80(05):759-762

6 Amon E, Lewis SV, Sibai BM, Villar MA, Arheart KL. Ampicillin prophylaxis in preterm premature rupture of the membranes: a prospective randomized study. Am J Obstet Gynecol 1988;159 (03):539-543
7 Ovalle A, Martínez MA, Kakarieka E, et al. Antibiotic administration in patients with preterm premature rupture of membranes reduces the rate of histological chorioamnionitis: a prospective, randomized, controlled study. J Matern Fetal Neonatal Med 2002; 12(01):35-41

8 Macy E. Penicillin and beta-lactam allergy: epidemiology and diagnosis. Curr Allergy Asthma Rep 2014;14(11):476

9 Salkind AR, Cuddy PG, Foxworth JW. The rational clinical examination. Is this patient allergic to penicillin? An evidence-based analysis of the likelihood of penicillin allergy. JAMA 2001;285 (19):2498-2505

10 Rouse DJ, Hirtz DG, Thom E, et al; Eunice Kennedy Shriver NICHD Maternal-Fetal Medicine Units Network. A randomized, controlled trial of magnesium sulfate for the prevention of cerebral palsy. N Engl J Med 2008;359(09):895-905

11 Kenyon S, Boulvain M, Neilson JP. Antibiotics for preterm rupture of membranes. Cochrane Database Syst Rev 2010;(08):CD001058

12 Hopkins MK, Dotters-Katz S, Boggess K, Heine RP, Smid M. Perioperative antibiotic choice in labored versus unlabored Cesareans and risk of postcesarean infectious morbidity. Am J Perinatol 2018;35(02):127-133

13 Kenyon SL, Taylor DJ, Tarnow-Mordi W; ORACLE Collaborative Group. Broad-spectrum antibiotics for preterm, prelabour rupture of fetal membranes: the ORACLE I randomised trial. Lancet 2001;357(9261):979-988

14 Weintraub AS, Ferrara L, Deluca L, et al. Antenatal antibiotic exposure in preterm infants with necrotizing enterocolitis. J Perinatol 2012;32(09):705-709

15 Silverman MA, Konnikova L, Gerber JS. Impact of antibiotics on necrotizing enterocolitis and antibiotic-associated diarrhea. Gastroenterol Clin North Am 2017;46(01):61-76

16 Munyaka PM, Eissa N, Bernstein CN, Khafipour E, Ghia JE. Antepartum antibiotic treatment increases offspring susceptibility to experimental colitis: a role of the gut microbiota. PLoS One 2015; 10(11):e0142536

17 Nogacka A, Salazar N, Suárez M, et al. Impact of intrapartum antimicrobial prophylaxis upon the intestinal microbiota and the prevalence of antibiotic resistance genes in vaginally delivered full-term neonates. Microbiome 2017;5(01):93

18 Hall CM, Milligan DW, Berrington J. Probable adverse reaction to a pharmaceutical excipient. Arch Dis Child Fetal Neonatal Ed 2004; 89(02):F184

19 Gershanik J, Boecler B, Ensley H, McCloskey S, George W. The gasping syndrome and benzyl alcohol poisoning. N Engl J Med 1982;307(22):1384-1388 